\title{
Anti-SLITRK6 Monoclonal Antibody-MMAE Conjugate AGS15E
}

National Cancer Institute

\section{Source}

National Cancer Institute. Anti-SLITRK6 Monoclonal Antibody-MMAE Conjugate AGS15E.

NCI Thesaurus. Code C111995.

An antibody-drug conjug ate (ADC) composed of a monoclonal antibody directed against

SLIT and NTRK-like protein 6 (SLIT RK6), covalently linked to the cytotoxic agent monomethyl auristatin $\mathrm{E}$ (MMAE), an auristatin derivative and a potent inhibitor of microtubule polymerization, with potential antineoplastic activity. Upon intravenous administration, the monoclonal antibody moiety of AGS15E binds to SLIT RK6 expressed on tumor cells, which facilitates both AGS15E internalization and the intracellular delivery of MMAE. Upon cleavage, MMAE binds to tubulin and inhibits its polymerization, which results in G2/M phase arrest and tumor cell apoptosis. SLITRK6, a member of the Slitrk family of leucine-rich repeat (LRR) neuronal transmembrane proteins, is minimally expressed in normal tissue but overexpressed in a variety of cancers, including bladder cancer, some forms of lung cancer, breast cancer and glioblastoma. 